\title{
Improvement of intestinal flora: accompany with the antihypertensive effect of electroacupuncture on stage 1 hypertension
}

\author{
Jun-meng Wang ${ }^{1 \dagger}$, Ming-xiao Yang ${ }^{2,3+}$, Qiao-feng Wu ${ }^{1,4^{*}} \mathbb{D}$, Ji Chen ${ }^{1}$, Shu-fang Deng ${ }^{1}$, Lin Chen ${ }^{1}$, Da-neng Wei \\ and Fan-rong Liang ${ }^{1 *}$
}

\begin{abstract}
Background: Increasing evidence have indicated the relationship between intestinal dysbiosis and hypertension. We aimed to evaluate the effect of the electroacupuncture $(E A)$ on intestinal microbiota in patients with stage 1 hypertension.

Methods: 93 hypertensive patients and 15 healthy subjects were enrolled in this study. Applying a highly accurate oscillometric device to evaluate the antihypertensive effect of EA. 16S rRNA sequencing was used to profile stool microbial communities from Healthy group, Before treatment (BT) group and After treatment (AT) group, and various multivariate analysis approaches were used to assess diversity, composition and abundance of intestinal microbiota.

Results: In this study, EA significantly decreased the blood pressure (BP) of hypertensive patients. Higher abundance of Firmicutes and lower Bacteroidetes abundance were observed in the BT group compared to the Healthy group. And EA treatment significantly decreased the Firmicutes/Bacteroidetes ratio compared to the BT group. Moreover, at the genus level, there was an increased abundance of Escherichia-Shigella in patients with hypertension, while Blautia were decreased, and EA reversed these changes.
\end{abstract}

Conclusions: Our study indicates that EA can effectively lower BP and improve the structure of intestinal microbiota which are correlate with the alteration of blood pressure by electroacupuncture.

Trial registration: Clinicaltrial.gov, NCT01701726. Registered 5 October 2012, https:/clinicaltrials.gov/ct2/show/study/ NCT01701726

Keywords: Intestinal flora, Electro-acupuncture, Stage 1 hypertension, Dysbiosis

\section{Background}

Hypertension is China's leading cause of cardiovascular diseases (CVD), including stroke, heart failure, coronary heart disease [1]. Because of the aging of the population and changes of life style and living environment,

*Correspondence: wuqiaofeng@cdutcm.edu.cn; acuresearch@126.com †Jun-meng Wang and Ming-xiao Yang contributed equally to this work ${ }^{1}$ Acupuncture and Moxibustion College, Chengdu University

of Traditional Chinese Medicine, No.37, Road Shi-Er-Qiao, Jinniu District, Chengdu 610075, Sichuan, China

Full list of author information is available at the end of the article hypertension is increasing in prevalence worldwide [2]. Many studies have shown Stage 1 hypertension (BP $140-159$ or $90-99 \mathrm{mmHg}$ ), also called mild hypertension [3], was significantly associated with a higher CVD risk and mortality across entire adulthood [4-7].Even among young adults, stage 1 hypertension before age 40 years also had significantly higher risk for subsequent cardiovascular disease events compared with those with normal blood pressure before age 40 years [8]. Therefore, early treatment of hypertension and its sequelae can greatly reduce the cardiovascular medical burden. However, current evidence demonstrated that antihypertensive drugs

(c) The Author(s) 2021. This article is licensed under a Creative Commons Attribution 4.0 International License, which permits use, sharing, adaptation, distribution and reproduction in any medium or format, as long as you give appropriate credit to the original author(s) and the source, provide a link to the Creative Commons licence, and indicate if changes were made. The images or other third party material in this article are included in the article's Creative Commons licence, unless indicated otherwise in a credit line to the material. If material is not included in the article's Creative Commons licence and your intended use is not permitted by statutory regulation or exceeds the permitted use, you will need to obtain permission directly from the copyright holder. To view a copy of this licence, visit http://creativeco mmons.org/licenses/by/4.0/. The Creative Commons Public Domain Dedication waiver (http://creativecommons.org/publicdomain/ zero/1.0/) applies to the data made available in this article, unless otherwise stated in a credit line to the data. 
used in the treatment of patients with stage 1 hypertension do not reduce mortality or morbidity but increase the risk of adverse events in RCTs $[9,10]$. As one of the nonpharmacologic treatments, acupuncture has been used to relieve abnormal blood pressure (BP) and its typical organ damages or symptoms for several decades. A recent meta-analysis showed that acupuncture could be used for treating hypertension, and it may have the same effects as common medication [11]. Other studies have also indicated that acupuncture is particularly effective to lower blood pressure in prehypertension and stage 1 hypertension [12, 13]. Hence, acupuncture could be a potential therapy for stage 1 hypertension.

Except for heredity and environment, intestinal microbiota has increasingly been recognized as a main risk factor for hypertension. Changes in composition and dysbiosis of intestinal microbiota were observed in multiple hypertensive patients and animals [14]. Researchers have identified some possible hypotheses linking dysbiosis to hypertension through indirect mechanism including the low-grade inflammation [15], increasing of gram-negative bacteria [16], reduction number of short-chain fatty acids (SCFAs) producing bacteria17, 18, generation of trimethylamine $\mathrm{N}$-oxide [19], and so on. However, few studies have explored the effect of acupuncture on the intestinal microbiota in patients with stage 1 hypertension. We therefore used high-throughput sequencing to determine changes in intestinal microbial community structure in patients received EA treatment. Our results provide new leads regarding the pathogenesis and treatment of hypertension.

\section{Methods \\ Participants}

A total of 108 subjects were enrolled:93 hypertensive patients and 15 healthy. All patients received EA treatment. Among them, 29 of whom had feces collected before and after treatment. 15 normal people were also collected feces after enrollment. The study protocol was approved by Sichuan Regional Ethical Review committee of the Teaching Hospital of Chengdu University of Traditional Chinese Medicine (2012KL-003) and conformed to the ethical guidelines of the 1975 Declaration of Helsinki. Patients of essential hypertension were included and clear diagnosis according to the seventh report of the joint national committee on prevention, detection, evaluation, and treatment of high blood pressure. Study participants were recruited from the third teaching hospital of Chengdu University of Traditional Chinese Medicine and its surrounding communities. Participants were enrolled if they fulfilled the following criteria: [1] aged between 45 and 75 years; [2] were diagnosed as stage 1 hypertension in the first visit, or used to be diagnosed as stage 1 hypertension in recent 1 year, but without any medication history; [3] without neurological, other cardiovascular, hepatic and renal disease, and other visceral diseases; [4] without any drugs or herbs in at 15 days before the start of the study; [5] did not participate in any other study; [6] agreed to cooperate with researchers in all research procedures after they were introduced this study; and [7] provided with written informed consent. Patients with any of the following conditions were excluded: [1] with hypertension which was secondary to other diseases, such as renal vascular disease, Cushing's syndrome, hyperadrenocorticism and drug-induced hypertension; [2] had complicated cardiovascular, digestive, respiratory, urinary, blood, nervous, endocrine system and other severe primary diseases and failed to effectively control in clinic; [3] accompanied by epilepsy, sleep apnea hypopnea syndrome, etc.; [4] with psychiatric symptoms such as severe depression or anxiety ( $S A S \geq 70$, or $S D S \geq 72$ ); [5] pregnant or lactating woman, or woman of reproductive age who was intended to conceive in recent 1 year; [6] with abnormality in laboratory test of blood biochemistry, or with contagious risks, such as HIV virus carrier, or patient with positive HBV superficial antigen; [7] with malignant tumor or other severe consuming diseases, or patient with infections or bleeding disorders; [8] alcoholics or drug abusers, or vegetarians; [9] used to suffer from acute diseases in recent 2 weeks, such as high fever, or gastritis; [10] used to administer any drug that may potentially impaired renal or hepatic function; or [11] undergoing other clinical trials.

\section{Interventions}

After inclusion, patients received 18 sessions of acupuncture treatment (3 times weekly for 6 weeks). Each session lasted $30 \mathrm{~min}$. We chose bilateral acupoints of taichong (LR3), taixi (KI3), renying (ST9), neiguan (PC6) according to traditional Chinese medicine theory and based on review of the literature. Disposable stainless-steel needles were used in acupuncture treatments. Insertion was followed by stimulation performed by lifting and thrusting the needle combined with twirling and rotating the needle sheath to produce the sensation known as deqi [20]. In addition, auxiliary acupuncture needles were inserted $2 \mathrm{~mm}$ lateral to each acupoint to a depth of $2 \mathrm{~mm}$ without manual stimulation. This method could ensure the electrical stimulation working on the local points. The HANS acupoint nerve stimulators (Model LH 200A; HANS Therapy Co) were used after needle insertion. The stimulation was $2 \mathrm{~Hz}$ continuous wave; intensity varied from 0.1 to $2.0 \mathrm{~mA}$ until patients felt comfortable. Acupuncture was performed by licensed acupuncturists with more than 5 years' experience. 
$24 \mathrm{~h}$ ambulatory blood pressure monitoring/Ambulatory blood pressure monitoring

$24 \mathrm{~h}$ ambulatory blood pressure recordings were obtained by a highly accurate oscillometric device (TM-2430, A\&D Instruments, Japan) within $24 \mathrm{~h}$ before acupuncture treatment and the second day of the final treatment respectively. The TM-2430 monitor measures blood pressure automatically, on the same principle as the conventional mercury sphygmomanometer, with a cuff and a microphone. The interval between measurements can be preselected, and BP readings are recorded on a data processor. Blood pressure measurements were taken automatically every $15 \mathrm{~min}$ from $8 \mathrm{AM}$ to $10 \mathrm{PM}$ and every $30 \mathrm{~min}$ from $10 \mathrm{PM}$ to $8 \mathrm{AM}$. If a reading were considered faulty by the device, it was programmed to remeasure, which helped to avoid missing a measurement. The data from the device were then downloaded onto a computer for subsequent analysis. Before the study started, the investigators were trained for the appropriate use of the BP measurement device used in the study, and had to demonstrate proficiency according to the manufacturer's instruction.

\section{Fecal microbial DNA extraction}

A total 73 Stool samples were collected, of which 15 samples were from healthy, 29 samples were collected before acupuncture treatment and 29 samples were collected at the second day of the final treatment. Fecal genomic DNA was isolated from stool samples by using a QIAamp DNA Stool Mini Kit (51504, Qiagen, German) according to the kit protocol with modifications. The quality of extracted genomic DNA was checked by using the $2 \%$ agarose gel, and the DNA concentrations were determined with QuantiFluor ${ }^{\mathrm{TM}}-\mathrm{ST}$ Handheld Fluorometer with Blue Channels (Promega, USA). The extracted DNA was stored at $-20^{\circ} \mathrm{C}$.

\section{Fecal bacterial identification}

Bacteria within each sample were identified via 16S rRNA Sequencing. PCR amplification for the V4 hypervariable regions of the bacterial $16 \mathrm{~S}$ rRNA gene was performed using the 515F forward (5'-GTGCCAGCMGCCGCG GTAA-3') and 806R reverse (5'-GGACTACHVGGG TWTCTAAT-3') primers. Nextera XT V2 index kit Sets $\mathrm{A}$ and $\mathrm{B}$ were used to barcode PCR products and the AMPure XP bead system (Illumina, USA) was used for purification. Barcoded DNA underwent quantification, normalization and pooling to develop sequencing libraries which were then sequenced on the Illumina MiSeq platform (Illumina, USA). The Quantitative insights Into Microbial Ecology (QIIME) v1.9.1 analysis tool was used to join and de-multiplex forward and reverse sequences.
Using the Silva reference database, the open reference operational taxonomic unit (OTU) picking method was used for taxonomic assignments with a pairwise identity threshold of $97 \%$. Prior to downstream analysis, the OTU table was rarefied to 3000 sequences/sample with no samples removed in this step. These OTUs were analyzed by global parameters (described below) including determination of diversity, evenness, richness, and relative abundance of the bacteria identified in each sample.

\section{Statistical analysis}

Paired t-test were performed to compare the baseline and post-treatment blood pressure. 16S rRNA gene sequencing data was analyzed on the free online platform of Majorbio Cloud Platform (www.majorbio.com). Chao1, Ace, Simpson and Shannon index were used for comparison of alpha diversity. Principle coordinates analysis (PCoA) (Bray-Curtis dissimilarity index) and partial least squares discriminant analysis (PLS-DA) were used to visualize the change of distribution patterns. The unpaired two-tailed $t$-test was used for comparisons between Healthy and Hypertension. The paired t-test was used for comparisons between BT group and AT group. Spearman's correlation coefficients were used to assess bivariate relationships. All results with $P<0.05$ between groups were considered statistically significant.

\section{Results}

\section{Baseline information}

Among 397 patients screened, 101 were enrolled at baseline. 8 patients never received a treatment and lost contact after baseline. A total of 93 patients received 18 sessions of EA treatment. The baseline characteristics and BP-related parameters such as gender, age, nationality, BMI, and other physiological parameters like blood sugar, total cholesterol, RBC, WBC demonstrated no significant difference in the hypertensive patients and healthy control. However, the systolic blood pressure (SBP) and diastolic blood pressure (DBP) were higher in the patients with hypertension, as shown in Table 1.

\section{EA treatment reduced blood pressure indices compared with the baseline}

After the six-week EA treatment, the blood pressure of patients differed significantly (Table 2). SBP (including daytime systolic blood pressure, dSBP and nighttime systolic blood pressure, nSBP) indices were significantly decreased more than $3 \mathrm{mmHg}$ after EA treatment compared to that in the baseline $(P<0.001, P<0.01, P<0.05)$. While DBP and dDBP (daytime diastolic blood pressure) decreased significantly after EA treatment $(P<0.05$, $P<0.05)$. nDBP (nighttime diastolic blood pressure) 
Table 1 Baseline characteristics of the study population

\begin{tabular}{lllr}
\hline Characteristic & Patients & Healthy & P value \\
\hline Age, mean (SD),y & $61.43(4.37)$ & $56.33(7.73)$ & 0.299 \\
Female (\%) & $68.80 \%$ & $80.00 \%$ & 0.291 \\
BMl, mean (SD) & $24.87(4.95)$ & $24.29(0.71)$ & 0.169 \\
Pulse, mean (SD) & $72.65(7.57)$ & $71.57(7.07)$ & 0.265 \\
Blood sugar (mmol/L) & $5.04(0.30)$ & $4.99(0.42)$ & 0.752 \\
Total Cholesterol (mmol/L) & $4.82(0.55)$ & $4.52(0.41)$ & 0.149 \\
RBC count (× 10 $12 / L)$ & $4.48(0.48)$ & $4.49(0.57)$ & 0.958 \\
WBC count (× 10 $/ \mathrm{L})$ & $5.13(0.93)$ & $5.18(1.41)$ & 0.917 \\
SBP, mean (SD) $\mathrm{mmHg}$ & $144.69(9.29)$ & $113.60(9.00)$ & $<0.0001$ \\
DBP, mean (SD) $\mathrm{mmHg}$ & $88.61(8.15)$ & $75.14(5.98)$ & $<0.0001$ \\
\hline
\end{tabular}

Demographic information and blood pressure indices. $B M I$ body mass index, $R B C$ red blood cell, $W B C$ white blood cell, SBP/DBP systolic/diastolic blood pressure, $P<0.05$ were considered statistically significant

did not show any difference before and after treatment $(P=0.051)$.

Alpha diversity showed no significant difference between Healthy group, BT group and AT group, whereas the three groups were clearly separated by PLS-DA.

In the current study, 73 samples were sequenced and a total of 3,440,986 high-quality raw sequences were generated. The average sequence length was 433 (min: 326, max: 458). Each sample was normalized to an equal sequencing depth and clustering. After filtering the low abundant $(<0.1 \%)$ OTUs, 870 OTUs were obtained at a $97 \%$ similarity level, from which 16 phyla, 30 classes, 55 orders, 98 families, 294 genera and 554 species were detected. Good's coverage was $99.82 \%$, indicating the sequences identified in this study could represent the majority of bacterial sequences present in the samples. Although rarefaction curves of numbers of observed OTUs per sample suggested new bacteria would be expected with additional sequencing (Additional file 1: Fig. S1a), the rarefaction curves for the Shannon diversity index for each sample reached plateaus, indicating that the majority of the diversity was already procured (Additional file 1: Fig. S1b).
There was no difference in the alpha diversity respect determined by and Simpson/Shannon (species diversity) and Chao1/Ace (species richness) index between the Healthy group, BT group and AT group (Fig. 1). No difference among three groups was found in the beta diversity with unsupervised PCoA (Additional file 2: Fig. S2). However, supervised PLS-DA showed clear separation between Healthy and hypertension patients although the microbiome of AT group was similar to that of the BT group (Fig. 2).

\section{EA treatment improved intestinal microbiota structure in patients with stage 1 hypertension}

A Venn diagram was used to show the number of common and unique OTUs, thus intuitively describing sample similarity and overlap. The unique OTUs in the Healthy, BT, and AT groups were 10, 75 and 12 respectively. The common OTU number in three groups was 570 , accounted for $92.08 \%, 68.75 \%$ and $74.51 \%$ of the total number of OTUs in the Healthy group (619), BT group (829) and AT group (765) (Fig. 3a). 5 phyla were identified with relative abundance more than $1 \%$. The bacteria analysis showed that the relative abundance of predominant phyla in Healthy group, BT group and AT group varies widely although the species was similar (Fig. $3 \mathrm{~b}$ ). Figure $3 \mathrm{c}$ showed differences in the species and relative abundance of the dominant genus.

At the phylum level (Fig. 4b), the relative abundances of the dominant phyla such as Firmicutes and Bacteroidetes were showed significant dissimilarities among three groups. Higher abundance of Firmicutes $(P<0.05)$ and lower Bacteroidetes abundance $(P<0.05)$ were observed in the BT group compared to Healthy group. And EA treatment significantly decreased the relative abundance of Firmicutes $(P<0.01)$ compared to BT group. Moreover, compared to Healthy group, the ratio of Firmicutes to Bacteroidetes was significantly increased in the BT group $(P<0.001)$ and reduced in acupuncture group $(P<0.05)$ (Fig. 4a).

Table 2 Outcome measurements during the study

\begin{tabular}{lcccc}
\hline Characteristic & Baseline & After treatment & MD (95\% Cl) & $P$ value \\
\hline SBP, mean (SD) mmHg & $144.69(9.29)$ & $140.48(9.77)$ & $4.36(1.97,6.74)$ & $2.16(0.45,3.87)$ \\
DBP, mean (SD) mmHg & $88.61(8.15)$ & $86.31(8.02)$ & $4.48(1.92,7.04)$ & $<0.001$ \\
dSBP, mean (SD) mmHg & $147.32(9.88)$ & $143.02(10.47)$ & $2.12(0.38,3.87)$ & $<0.05$ \\
dDBP, mean (SD) mmHg & $90.30(8.49)$ & $88.10 .23(8.24)$ & $3.77(0.91,6.64)$ & $<0.01$ \\
nSBP, mean (SD) mmHg & $136.20(12.21)$ & $132.59(12.90)$ & $2.10(-0.01,4.21)$ & $<0.05$ \\
nDBP, mean (SD) mmHg & $82.83(8.78)$ & $80.60(9.64)$ & 0.05 \\
\hline
\end{tabular}

Blood pressure changes after six-week EA treatment. $M D$ mean difference, $d S B P / d D B P$ daytime systolic/diastolic blood pressure, $n S B P / n D B P$ nighttime systolic/diastolic blood pressure. All data were analyzed by paired-sample $T$ test, $P<0.05$ were considered statistically significant 

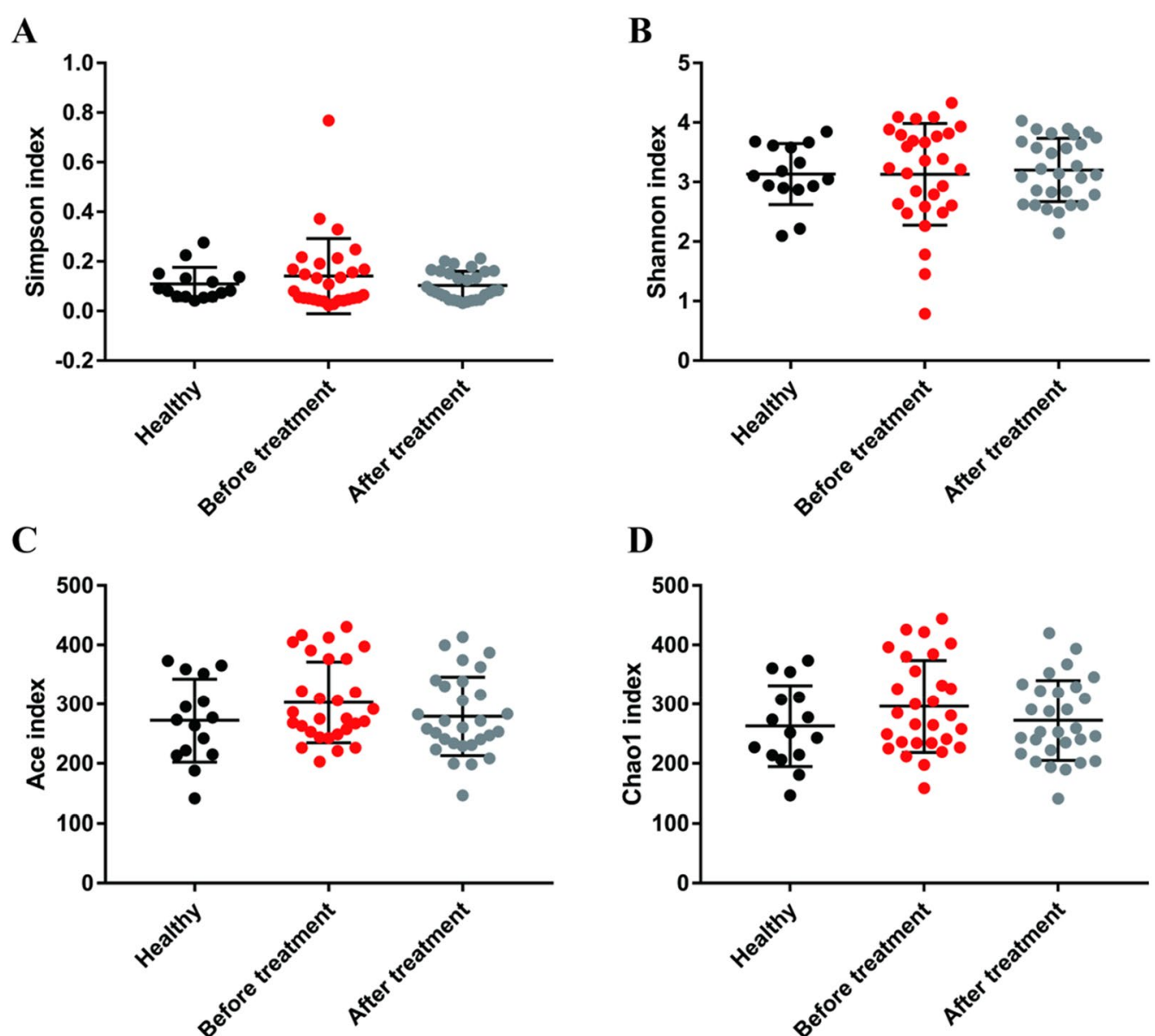

Fig. 1 Influence of EA on the diversity and richness of gut microbiota. a Simpson and $\mathbf{b}$ Shannon index represent community diversity of OTUs. $\mathbf{c}$ Ace and $\mathbf{d}$ Chao 1 index represent community richness of OTUs. There was no significant difference in Alpha diversity among three groups

At the Family level (Fig. 4c), differences were observed in the Bifidobacteriaceae and Lachnospiraceae. Bifidobacteriaceae in BT group decreased compared to the Healthy group $(P<0.05)$. In addition, lower Lachnospiraceae abundance were observed in the Healthy group compared to the BT group $(P<0.05)$.

At the genus level (Fig. $4 \mathrm{~d}$ ), differences were observed in the Blautia and Escherichia-Shigella. The relative abundance of Blautia in the BT group was significantly decreased compared to the Healthy group $(P<0.01)$, but increased after EA treatment $(P<0.05)$. In contrast, BT group showed greater abundance of EscherichiaShigella than that in the Healthy group $(P<0.05)$ and EA treatment $(P<0.05)$ decreased the abundance of Escherichia-Shigella.

\section{Potential correlation between clinical parameters and microbiota}

We further assessed the correlation between the 10 most prevalent bacteria at family- and genus-level and blood pressure (Fig. 5). The $f_{-}$Streptococcaceae $(\mathrm{R}=0.27$, $P<0.05)$ and $g \_$Escherichia-Shigella $(\mathrm{R}=0.37, P<0.05)$ showed positive correlations with DBP. In addition, the negative correlations were observed for g_Lachnoclostridium $(\mathrm{R}=--0.31, P<0.05)$ and $g$ Eubacterium hallii $(\mathrm{R}=-0.32, P<0.05)$ with SBP. Moreover, g_Blautia were simultaneously found significant negative correlation with $\mathrm{SBP}(\mathrm{R}=-0.37, P<0.05)$ and $\mathrm{DBP}(\mathrm{R}=-0.40$, $P<0.01)$.

\section{Discussion}

There is convincing evidence suggesting that balance of intestinal microbiota is an important factor in hypertension although the mechanism is complex and in part unknown [21-23]. In this study, we investigated the antihypertensive effect of EA treatment in patients with stage 1 hypertension. The Firmicutes and Bacteroidetes changed in the patients before treatment, while EA significantly reduced Firmicutes abundance and the ratio of Firmicutes to Bacteroidetes. Moreover, EA increased 


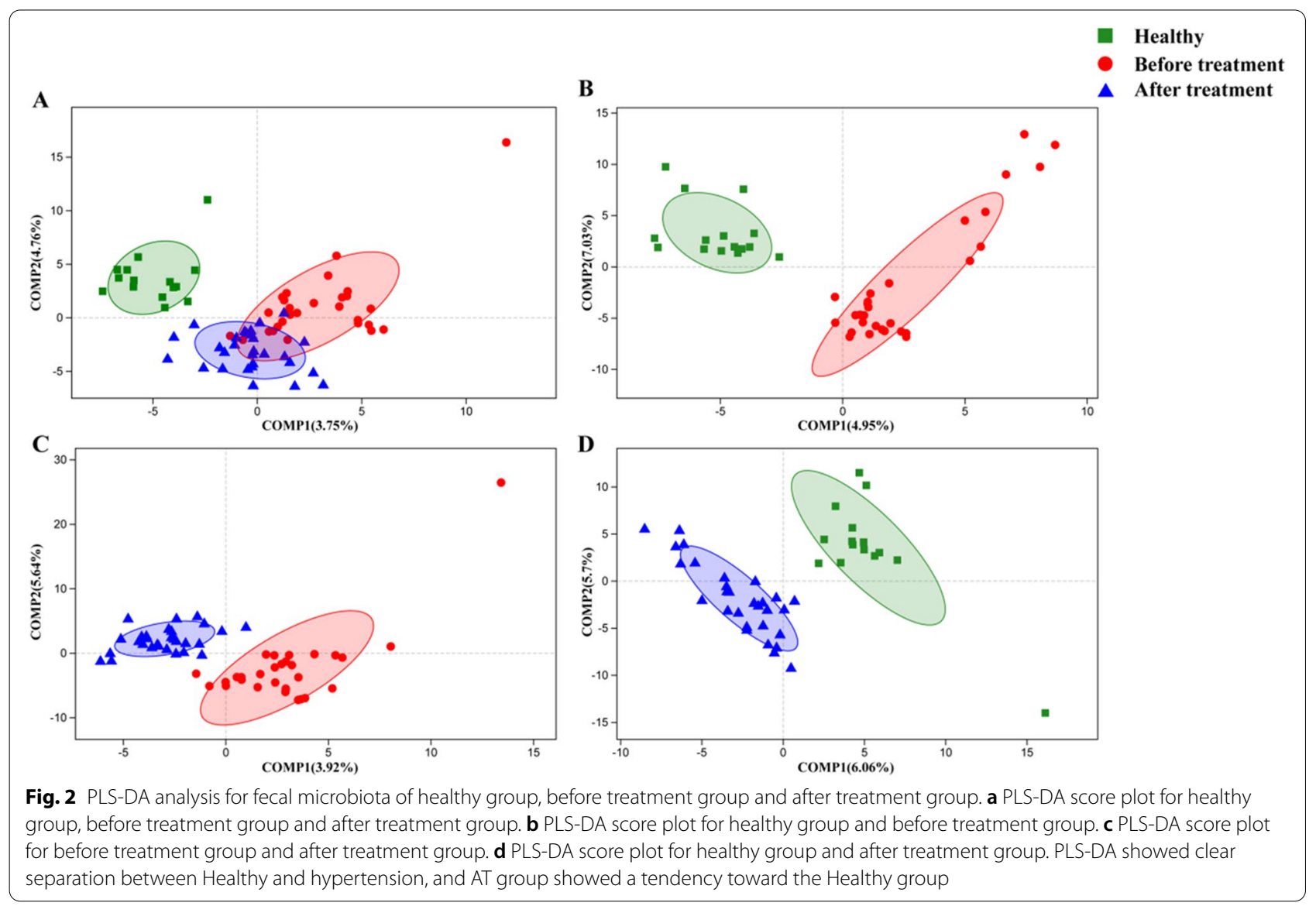

the relative abundances of Blautia and reduced that of Escherichia-Shigella compared with BT group. Therefore, we suggest that the mechanism of reducing blood pressure by EA might be closely related with structural changes of gut flora.

This study showed that EA treatment at the aucpoints of taichong (LR3), taixi (KI3), renying (ST9) and neiguan (PC6) significantly decreased the SBP $(-4.4 \mathrm{mmHg}$; $P<0.001)$ and DBP $(-2.16 \mathrm{mmHg} ; P<0.05)$. This finding was similar with a randomized, controlled, assessorblinded pilot trial to assess the effects of acupuncture on prehypertension and stage 1 hypertension, in which SBP was reduced $5.7 \mathrm{mmHg}$ [12]. Several high-quality evidences for patients with ungraded hypertension (SBP $\geq 140 \mathrm{mmHg}$ or $\mathrm{DBP} \geq 90 \mathrm{mmHg}$ ) also showed the approximate range of $\mathrm{BP}$ reduction fluctuated [2427]. The reduction range of SBP and $\mathrm{nDBP}$ was 3.6$5.4 \mathrm{mmHg}$ and $3-7 \mathrm{mmHg}$, respectively. Previous studies have estimated the extent of protection when BP is lowered. Girerd, et al. [28] showed that each $2 \mathrm{mmHg}$ reduction in SBP was associated with a $25 \%$ reduction in stroke events. A reduction in SBP by at least $5 \mathrm{~mm} \mathrm{Hg}$ in the general population was corresponding to a $9 \%$ reduction in mortality caused by coronary heart disease, and a 7\% reduction in all-cause mortality [29, 30]. Therefore, EA has positive clinical meaningful effect on patients with stage 1 hypertension.

In addition, our data showed that EA decreased the ratio of Firmicutes to Bacteroidetes. In the healthy intestine, Bacteroidetes and Firmicutes contribute most types of the total bacterial species, and their ratio is often considered as a relative measurement of intestinal microbial health or dysbiosis [31]. The alteration of Firmicutes/Bacteroidetes ratio has been observed in multiple animal models of hypertension [22, 32, 33]. A pioneer study in the field compared the composition of microbiota between spontaneously hypertensive rats, chronic angiotensin II (Ang II) infusion rats and Wistar Kyoto (WKY) control rats. The results showed the Firmicutes/Bacteroidetes ratio in these models was 5 and 3 times that of the control group, respectively [21]. Same results were validated in spontaneously hypertensive stroke prone rats (SHRSP) [34]. In the experiment, SHRSP and WKY rats were performed fecal transplants after they were treated with broad-spectrum antibiotics. Ten weeks later, the WKY rats transplanted with caecum 

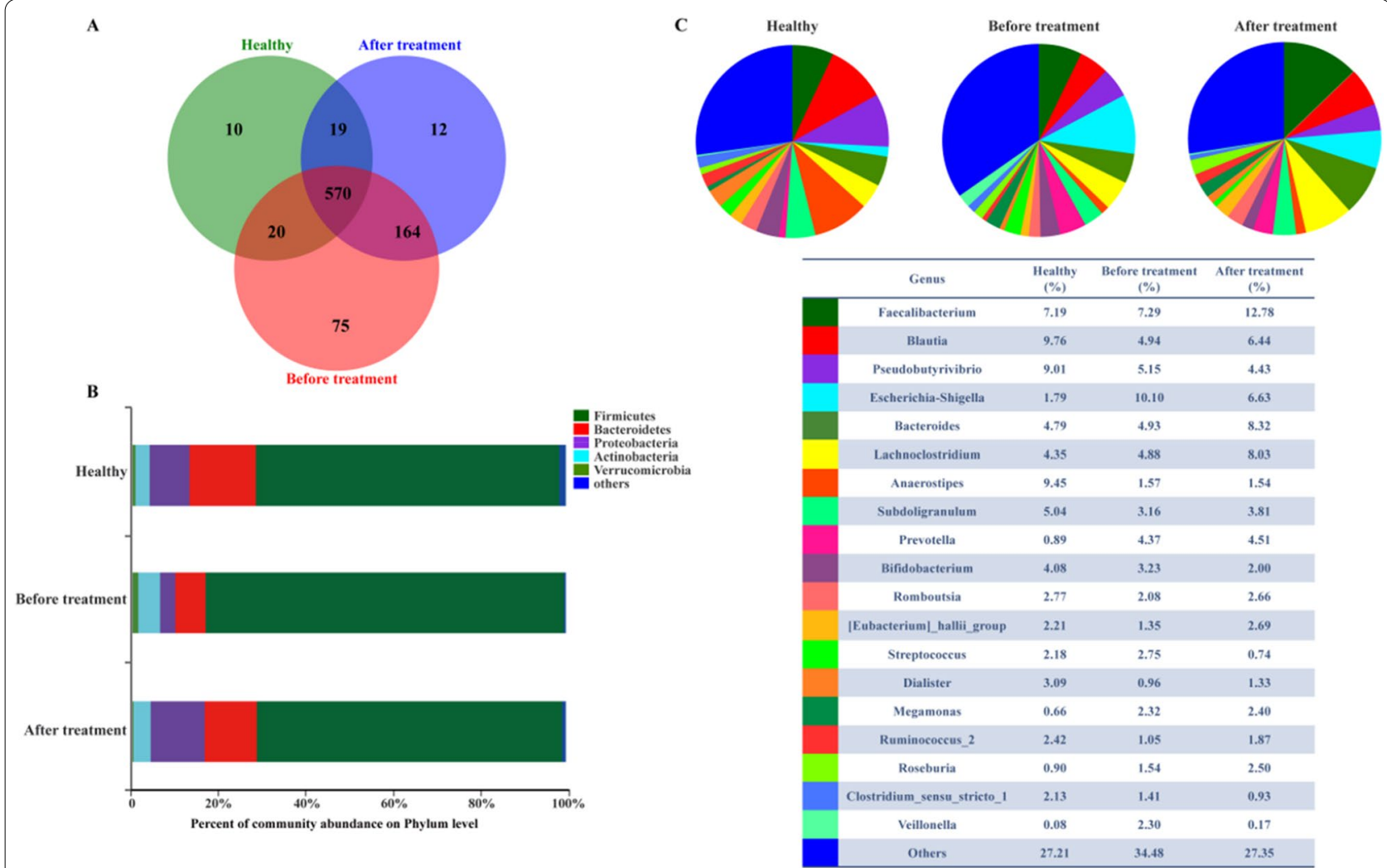

Fig. 3 Modulation of the composition of gut microbiota by EA. a Venn diagrams demonstrate the number of OTUs shared between the Healthy, BT and AT group by the overlap. b Bar chart depicts the variability in phylum-level composition in each group. $\mathbf{c}$ Pie chart shows the proportion of reads in genus for the Healthy, BT and AT group

content from SHRSP showed significant increasing in the $\mathrm{BP}$ and Firmicutes/Bacteroidetes ratio, confirming the contribution of intestinal flora to hypertension. In the following years, similar results were obtained with others hypertensive animal models (i.e., obstructive sleep apneainduced hypertensive rats; Dahl salt-sensitive and saltresistant rats; deoxycorticosterone acetate (DOCA)-salt hypertensive mice) [35-37]. In our cohort, a significant higher abundance of Firmicutes and a lower abundance of Bacteroidetes were exhibited in individuals with stage 1 hypertension compared to healthy group. These findings were in agreement with the findings of Nosheen et al., who have demonstrated higher Firmicutes/Bacteroidetes ratio in patients with grade 3 hypertension [23]. Thus, the results indicated that intestinal dysbiosis in early stage promotes the development of hypertension, and EA may play a role in lowering blood pressure accompanying with improving gut dysbiosis.

Moreover, we observed the regulation of EA on Blautia and Escherichia-Shigella at genus level. Blautia is a group of bacteria that produces multiple SCFAs, including acetate [38], propionate [39], succinate and lactate in some subspecies [40]. SCFAs are a major class of bacterial metabolites and are widely recognized as potential mediators involved in regulating $\mathrm{BP}$ and inhibiting chronic inflammation [17, 41]. Acetate has been shown to reduce systolic and diastolic blood pressure, cardiac fibrosis and left ventricular hypertrophy in DOCA-salt hypertensive mice [37]. Another study reported that propionate reduced BP and attenuated Ang II-induced cardiac hypertrophy and fibrosis by mitigating systemic inflammation [17]. In addition, SCFAs may exert their effects by regulating renin release and vasomotor function via olfactory receptor and endothelial $G$ proteincoupled receptor [42-44]. Lower abundance of Blautia has been detected in hypertensive patients with preeclampsia compared to healthy, pregnant control subjects [45], which was in agreement with our observation. The abundance of Blautia was also negatively correlated with systolic BP in spontaneously hypertensive rats and healthy male volunteers $[46,47]$. In the present study, the significant increase of Blautia in EA group may be closely related to decreased blood pressure. Escherichia-Shigella is a group of gram-negative bacteria containing lipopolysaccharides (LPS) in their cell walls [22]. It is known that LPS activates toll-like receptor 4 (TLR4) pathway [48], 

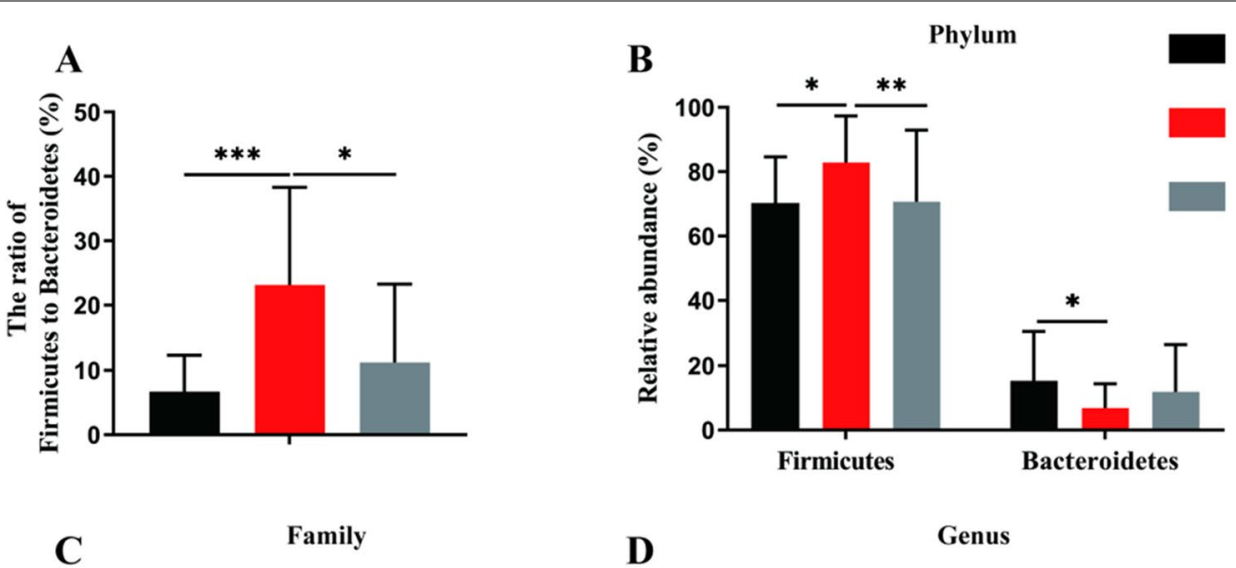

Healthy
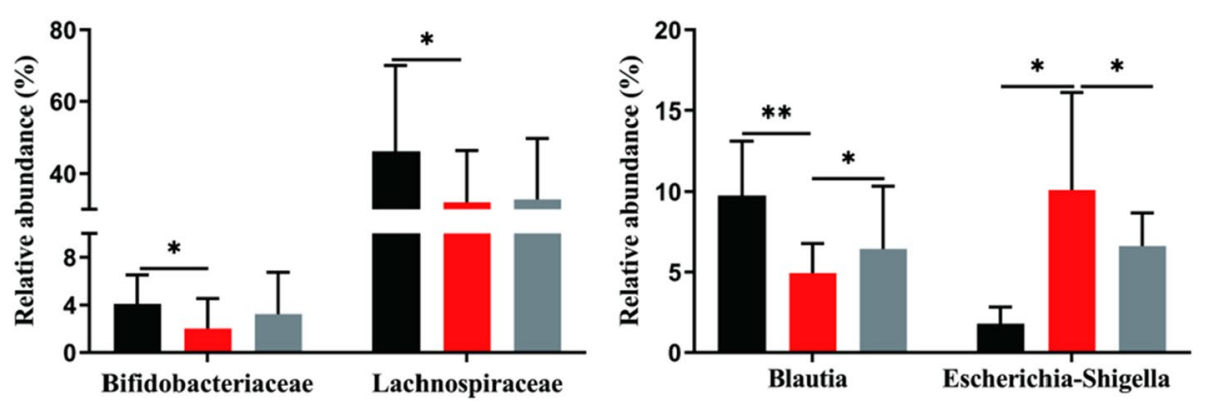

Fig. 4 Influence of EA on the relative abundance of microbiota at each level. a The ratio of Firmicutes to Bacteroidetes ratio, and $\mathbf{b}-\mathbf{d}$ differences at each taxonomic level (phylum, family and genus). All data were expressed as mean \pm SD. ${ }^{*} P<0.05$, ${ }^{* *} P<0.01,{ }^{* * *} P<0.001$

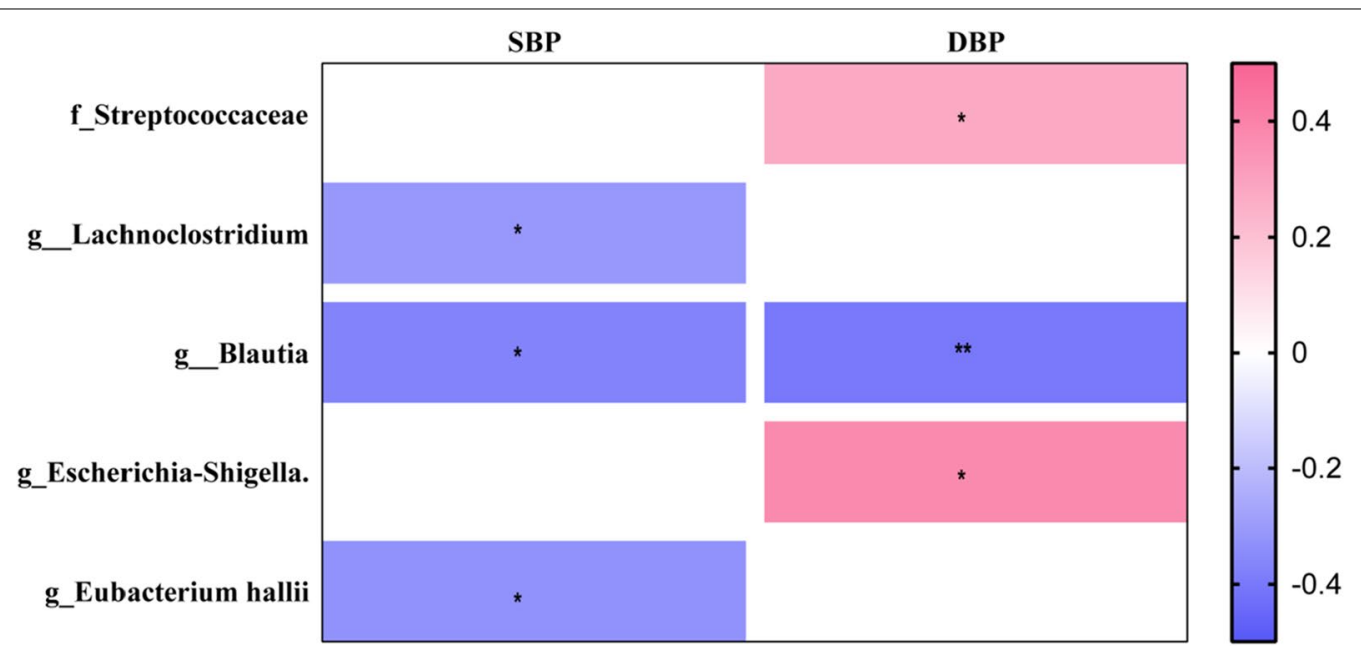

Fig. 5 Spearman correlation plots between bacterial at family and genus level and BP values. R in different colors to show, the right side of the legend is the color range of different $R$ values. ${ }^{*} P<0.05,{ }^{* *} P<0.01$

which in turn induced the activation of proinflammatory cytokines (TNF- $\alpha$, IL-6, and IL-1) and further led to inflammatory [49-51]. Bomfim and colleagues [22, 52] described that the inhibition of TLR4 activation by chronic treatment with anti-TLR4 antibody reduced both
BP and endothelial dysfunction in spontaneously hypertensive rats. These results were in concordance with finding in Ang II-induced mice [53]. In this study, reduced level of Escherichia-Shigella after EA treatment may suggest an improvement inflammatory status of the host. 
We also identified a significant correlation between BP values and 5 microbial taxa in the family at genus level. F_Streptococcaceae,g_Eubacterium hallii and g_Lachnoclostridium were observed correlation with blood pressure. Gut Streptococcaceae is also associated with inflammatory bowel disease [54] and liver cirrhosis [55]. Moreover, a study has showed that Streptococcus from gut might involve in the formation of atherosclerotic plaque microbiota [56]. Eubacterium hallii is a common member of the adult gut microbiota that can produce butyrate from lactate and acetate, and convert 1,2-propanediol to propionate $[57,58]$. Udayappan, et al. [59] demonstrated that feeding Eubacterium hallii increased energy expenditure and fecal butyrate concentrations, thus modifying bile acid metabolism of $\mathrm{db} / \mathrm{db}$ mice. Lachnoclostridium, a species belonging to Lachnospiraceae, was also a butyrate-producing bacterium [60], which can bind to metabolite-sensing G-protein coupled receptors (GPCRs) to regulate blood pressure via controlling gut homeostasis, host metabolism, and immune response [13].

In addition, we noticed an interesting phenomenon that hypertensive patients seem to produce more types of bacteria, although the abundance of these bacteria is very low. Further analysis showed that most of these bacteria belong to Ruminococcaceae, Clostridiales (Additional file 3: Table. S1). A phenome-wide association study has demonstrated the association between genus Ruminococcus and hypertension [61]. Another report shows that multiple species of Clostridiales (e.g., Ruminococcaceae, Family_XIII, etc.) were higher in non-treated hypertensive (SBP:153.1 \pm 14.6$)$ than normotensive (SBP:109.7 \pm 7.1 ) [62]. An animal experiment also confirmed that high-carbohydrate, high-fat diet induced increasing Clostridia in hypertensive rats in the colonic microbiota [63]. Therefore, types of bacteria, especially Ruminococcaceae and Clostridiales should be enough attention in hypertensive patients.

As to the underlying mechanism or the target of acupuncture, neuroendocrine immunity system may be considered as an important pathway. As we mentioned, the association between intestinal microbiota and the host are complex and bi-direction. On the one hand, intestinal bacteria can modulate the host by producing a wide range of metabolites (SCFAs, bile acids, tryptophan et al.) and by their own component. Some of these metabolites and the bacterial components are risk markers for hypertension, inflammation and metabolic syndrome. On the other hand, the metabolism of host can regulate the microbiota in gut. For instance, the colonocyte metabolism works as a control switch, mediating a shift between homeostatic and dysbiosis communities [31]. Also, some of the metabolites such as butyrate activates PPAR $\gamma$ signaling in human epithelial cells [64] can drive the metabolism of surface colonocytes towards mitochondrial $\beta$-oxidation of fatty acids [65-67], in turn maintain epithelial hypoxia [68]. Therefore, it's hard to say which of these two aspects (the flora and the host) is the direct target of acupuncture. A recent review [69] summarized the mechanism of acupuncture in treating hypertension and found that renin-angiotensin-aldosterone system (RAAS), vascular endothelium, oxidative stress response, neuroendocrine system are all involved in the antihypertensive mechanisms of acupuncture while these mechanisms themselves are influenced by gut microbiota. In addition, previous studies showed EA could stimulate more SCFAs and improve the digestion. Therefore, further studies are needed to establish the contribution of specific bacteria controlled by EA to blood pressure regulation.

There were several limitations in this study. Firstly, we did not observe the long-term effect of EA on hypertension and intestinal microbiota. A study has reported that the antihypertensive effect of acupuncture could be decreased with the elapse of time [25]. Another limitation is that the gut microbial community would be sensitive to environmental factors [70], larger cohort containing multi-types of hypertensive patients are needed for further investigation.

\section{Conclusion}

The present study shows that electroacupuncture can effectively lower blood pressure and improve the structure of intestinal microbiota which are correlate with the alteration of blood pressure by EA. These findings could inspire future research for understanding the mechanism underlying the therapeutic effects of acupuncture on hypertension.

\section{Supplementary Information}

The online version contains supplementary material available at https://doi. org/10.1186/s13020-020-00417-8.

Additional file 1: Fig. S1 Alpha diversity rarefaction curves of samples based on species-level OTUs.

Additional file 2: Fig. S2 Analysis of principal coordinate analysis (PCOA) with the bray_curtis distance matrix on the OTU level.

Additional file 3: Table S1. Unique OTUs in BT group.

Abbreviations

EA: Electroacupuncture; BT: Before treatment; AT: After treatment; CVD: Cardiovascular diseases; BP: Blood pressure; SCFAs: Short-chain fatty acids; Ang II: Angiotensin II; WKY: Wistar Kyoto rat; SHRSP: Spontaneously hypertensive stroke prone rats; LPS: Lipopolysaccharides; TLR4: Toll-like receptor 4; GPCRs: G-protein coupled receptors; PVN: Paraventricular nucleus; ROS: Reactive oxygen species.

Acknowledgements

Not applicable. 


\section{Authors' contributions}

Conceptualization, QW and FL; Funding acquisition, FL and QW; Formal analysis, JW and LC; Investigation, JW, MY and JC; Methodology, JW and SD; Project administration, QW and FL; Visualization, JW and DW; Writing Original Draft Preparation, JW and MY; Writing Review \& Editing, QW. All authors read and approved the final manuscript.

\section{Funding}

This study was financially supported by the National Natural Science Foundation of China (No.81590950, 81873383), the National Basic Research Program of China (No.2012CB518501), Fund of Science and Technology Department of Sichuan Province, China (No. 18YYJC0095, 2018JY0482) and Fund of Sichuan Provincial Department of Education, China (No. 18TD0018).

\section{Availability of data and materials}

The datasets used and/or analysed during the current study are available from the corresponding author on reasonable request.

\section{Ethics approval and consent to participate}

The study protocol was approved by Sichuan Regional Ethical Review committee of the Teaching Hospital of Chengdu University of Traditional Chinese Medicine(2012KL-003) and conformed to the ethical guidelines of the 1975 Declaration of Helsinki.

\section{Consent for publication}

Not applicable.

\section{Competing interests}

The authors declare that they have no competing interests.

\begin{abstract}
Author details
${ }^{1}$ Acupuncture and Moxibustion College, Chengdu University of Traditional Chinese Medicine, No.37, Road Shi-Er-Qiao, Jinniu District, Chengdu 610075, Sichuan, China. ${ }^{2}$ School of Chinese Medicine, The University of Hong Kong, 10 Sassoon Road, Pokfulam, Hong Kong, SAR, China. ${ }^{3}$ Department of Chinese Medicine, The University of Hong Kong-Shenzhen Hospital, Hai yuan Road, Futian District, Shenzhen 518053, Guangdong, China. ${ }^{4}$ Institute of Acupuncture and Homeostasis Regulation, Chengdu University of Traditional Chinese Medicine, Chengdu 610075, Sichuan, China.
\end{abstract}

Received: 17 May 2020 Accepted: 22 December 2020

Published online: 07 January 2021

\section{References}

1. Wang Z, Chen Z, Zhang L, Wang X, Hao G, Zhang Z, et al. Status of hypertension in China: results from the China hypertension survey, 2012-2015. Circulation. 2018;137(22):2344-56.

2. Bhatt DL, Kandzari DE, O'Neill WW, D'Agostino R, Flack JM, Katzen BT, et al. A controlled trial of renal denervation for resistant hypertension. N Engl J Med. 2014;370(15):1393-401.

3. Viera AJ, Hawes EM. Management of mild hypertension in adults. BMJ. 2016. https://doi.org/10.1136/bmj.i5719.

4. Ettehad D, Emdin CA, Kiran A, Anderson SG, Callender T, Emberson $J$, et al. Blood pressure lowering for prevention of cardiovascular disease and death: a systematic review and meta-analysis. Lancet. 2016;387(10022):957-67.

5. Cho L, Kim P. 2017 ACC/AHA blood pressure classification and cardiovascular disease in 15 million adults of age 20-94 years. J Clin Med. 2019;8(11):1832.

6. Liu N, Yang JJ, Meng R, Pan X-F, Zhang X, He M, et al. Associations of blood pressure categories defined by 2017 ACC/AHA guidelines with mortality in China: pooled results from three prospective cohorts. Eur J Prev Cardiol. 2017. https://doi.org/10.1177/2047487319862066.

7. Rapsomaniki E, Timmis A, George J, Pujades-Rodriguez M, Shah AD, Denaxas $\mathrm{S}$, et al. Blood pressure and incidence of twelve cardiovascular diseases: Lifetime risks, healthy life-years lost, and age-specific associations in 1.25 million people. Lancet. 2014;383(9932):1899-911.

8. Yano Y, Reis JP, Colangelo LA, Shimbo D, Viera AJ, Allen NB, et al. Association of blood pressure classification in young adults Using the 2017
American College of Cardiology/American Heart Association Blood Pressure Guideline With Cardiovascular Events Later in Life. JAMA. 2018;320(17):1774. https://doi.org/10.1001/jama.2018.13551.

9. Diao D, Wright JM, Cundiff DK, Gueyffier F. Pharmacotherapy for mild hypertension. Cochrane Database of Syst Rev. 2012. https://doi. org/10.1002/14651858.CD006742.pub2.

10. Sheppard JP, Stevens S, Stevens R, Martin U, Mant J, Hobbs FDR, et al. Benefits and harms of antihypertensive treatment in low-risk patients with mild hypertension. JAMA Intern Med. 2018;178(12):1626-34.

11. Tan X, Pan Y, Su W, Gong S, Zhu H, Chen H, et al. Acupuncture therapy for essential hypertension: a network meta-analysis. Ann Transl Med. 2019;7(12):266-266.

12. Liu Y, Park JE, Shin KM, Lee M, Jung HJ, Kim AR, et al. Acupuncture lowers blood pressure in mild hypertension patients: a randomized, controlled, assessor-blinded pilot trial. Complement Ther Med. 2015. https://doi. org/10.1016/j.ctim.2015.06.014.

13. Yang M, Yu Z, Chen X, Guo Z, Deng S, Chen L, et al. Active acupoints differ from inactive acupoints in modulating key plasmatic metabolites of hypertension: a targeted metabolomics study. Sci Rep. 2018;8(1):17824.

14. Kang Y, Cai Y. Gut microbiota and hypertension: From pathogenesis to new therapeutic strategies. Clin Res Hepatol Gastroenterol. 2018;42(2):110-7. https://doi.org/10.1016/j.clinre.2017.09.006.

15. Niskanen L, Laaksonen DE, Nyyssönen K, Punnonen K, Valkonen VP, Fuentes $R$, et al. Inflammation, abdominal obesity, and smoking as predictors of hypertension. Hypertension. 2004;44(6):859-65.

16. Li J, Zhao F, Wang Y, Chen J, Tao J, Tian G, et al. Gut microbiota dysbiosis contributes to the development of hypertension. Microbiome. 2017. https://doi.org/10.1186/s40168-016-0222-x.

17. Bartolomaeus H, Balogh A, Yakoub M, Homann S, Markó L, Höges S, et al. Short-chain fatty acid propionate protects from hypertensive cardiovascular damage. Circulation. 2019;139(11):1407-21.

18. Felizardo RJF, Mizuno Watanabe IK, Dardi P, Venturini Rossoni L, Olsen Saraiva Câmara N. The interplay among gut microbiota, hypertension and kidney diseases: the role of short-chain fatty acids. Pharmacol Res. 2019;141:366-77.

19. Zhu W, Gregory JC, Org E, Buffa JA, Gupta N, Wang Z, et al. Gut microbial metabolite TMAO enhances platelet hyperreactivity and thrombosis risk. Cell. 2016;165(1):111-24.

20. Zhao L, Li D, Zheng H, Chang X, Cui J, Wang R, et al. Acupuncture as adjunctive therapy for chronic stable angina. JAMA Intern Med. 2019;179:1-12.

21. Yang T, Santisteban MM, Rodriguez V, Li E, Ahmari N, Carvajal JM, et al. Gut dysbiosis is linked to hypertension. Hypertension. 2015;65(6):1331-40.

22. Sircana A, De Michieli F, Parente R, Framarin L, Leone N, Berrutti M, et al. Gut microbiota, hypertension and chronic kidney disease: recent advances. Pharmacol Res. 2019;144:390-408.

23. Mushtaq N, Hussain S, Zhang S, Yuan L, Li H, Ullah S, et al. Molecular characterization of alterations in the intestinal microbiota of patients with grade 3 hypertension. Int J Mol Med. 2019;44(2):513-22.

24. Macklin EA, Wayne PM, Kalish LA, Valaskatgis P, Thompson J, Pian-Smith MCM, et al. Stop Hypertension with the Acupuncture Research Program (SHARP): results of a randomized, controlled clinical trial. Hypertension. 2006;48(5):838-45.

25. Flachskampf FA, Gallasch J, Gefeller O, Gan J, Mao J, Pfahlberg AB, et al. Randomized trial of acupuncture to lower blood pressure. Circulation. 2007;115(24):3121-9.

26. Brook RD, Appel LJ, Rubenfire M, Ogedegbe G, Bisognano JD, Elliott WJ, et al. Beyond medications and diet: alternative approaches to lowering blood pressure: a scientific statement from the American Heart Association. Hypertension. 2013;61(6):1360-83.

27. Yin CS, Seo BK, Park HJ, Cho M, Jung WS, Choue R, et al. Acupuncture, a promising adjunctive therapy for essential hypertension: A doubleblind, randomized, controlled trial. Neurol Res. 2007. https://doi. org/10.1179/016164107X172220.

28. Endres M, Heuschmann PU, Laufs U, Hakim AM. Primary prevention of stroke: Blood pressure, lipids, and heart failure. Eur Heart J. 2011;32:545-55.

29. Whelton PK, He J, Appel $\sqcup$, Cutler JA, Havas S, Kotchen TA, et al. Primary prevention of hypertension: clinical and public health advisory from the National High Blood Pressure Education Program. J Am Med Assoc. 2002;288(15):1882-8. 
30. Zheng H, Li J, Li Y, Zhao L, Wu X, Chen J, et al. Acupuncture for patients with mild hypertension: a randomized controlled trial. J Clin Hypertens. 2019;21(3):412-20.

31. Tang WHW, Bäckhed F, Landmesser U, Hazen SL. Intestinal microbiota in cardiovascular health and disease: JACC state-of-the-art review. J Am Coll Cardiol. 2019. https://doi.org/10.1016/j.jacc.2019.03.024.

32. Kim S, Goel R, Kumar A, Qi Y, Lobaton G, Hosaka K, et al. Imbalance of gut microbiome and intestinal epithelial barrier dysfunction in patients with high blood pressure. Clin Sci. 2018;132(6):701-18.

33. Jama HA, Kaye DM, Marques FZ. The gut microbiota and blood pressure in experimental models. Curr Opin Nephrol Hypertens. 2019;28:97-104.

34. Adnan S, Nelson JW, Ajami NJ, Venna VR, Petrosino JF, Bryan RM, et al. Alterations in the gut microbiota can elicit hypertension in rats. Physiol Genomics. 2017:49(2):96-104

35. Mell B, Jala VR, Mathew AV, Byun J, Waghulde H, Zhang Y, et al. Evidence for a link between gut microbiota and hypertension in the Dahl rat. Physiol Genomics. 2015;47(6):187-97.

36. Durgan DJ, Ganesh BP, Cope JL, Ajami NJ, Phillips SC, Petrosino JF, et al. Role of the gut microbiome in obstructive sleep apnea-induced hypertension. Hypertension. 2016;67(2):469-74

37. Marques FZ, Nelson E. High-fiber diet and acetate supplementation development of hypertension and heart failure in hypertensive mice. Circulation. 2017; 135:964-77.

38. Martínez I, Lattimer JM, Hubach KL, Case JA, Yang J, Weber CG, et al. Gut microbiome composition is linked to whole grain-induced immunological improvements. ISME J. 2013;7(2):269-80.

39. Reichardt N, Vollmer M, Holtrop G, Farquharson FM, Wefers D, Bunzel M, et al Specific substrate-driven changes in human faecal microbiota composition contrast with functional redundancy in short-chain fatty acid production. ISME J. 2018;12(2):610-22. https://doi.org/10.1038/ismej.2017.196.

40. Liu C, Finegold SM, Song Y, Lawson PA. Reclassification of Clostridium coccoides, Ruminococcus hansenii, Ruminococcus hydrogenotrophicus, Ruminococcus luti, Ruminococcus productus and Ruminococcus schinkii as Blautia coccoides gen. nov., comb. nov., Blautia hansenii comb. nov., Blautia hydrogenotrophica comb. nov., Blautia luti comb. nov., Blautia producta comb. nov., Blautia schinkii comb. nov. and description of Blautia wexlerae. Int I Syst Evol Microbiol. 2008:58(8):1896-902.

41. Kelly CJ, Zheng L, Campbell EL, Saeedi B, Scholz CC, Bayless AJ, et al. Crosstalk between microbiota-derived short-chain fatty acids and intestinal epithelial HIF augments tissue barrier function. Cell Host Microbe. 2015;17(5):662-71.

42. Pluznick JL, Protzko RJ, Gevorgyan H, Peterlin Z, Sipos A, Han J, et al. Olfactory receptor responding to gut microbiotaderived signals plays a role in renin secretion and blood pressure regulation. Proc Natl Acad Sci U S A. 2013;110(11):4410-5.

43. Natarajan N, Hori D, Flavahan S, Steppan J, Flavahan NA, Berkowitz DE, et al. Microbial short chain fatty acid metabolites lower blood pressure via endothelial G protein-coupled receptor 41. Physiol Genomics. 2016;48(11):826-34.

44. Pluznick JL. Microbial short-chain fatty acids and blood pressure regulation. Curr Hypertens Rep. 2017;19(4):25.

45. Chang Y, Chen Y, Zhou Q, Wang C, Chen L, Di W, et al. Short-chain fatty acids accompanying changes in the gut microbiome contribute to the development of hypertension in patients with preeclampsia. Clin Sci (Lond). 2020;134(2):289-302.

46. Queipo-Ortuño MI, Boto-Ordóñez M, Murri M, Gomez-Zumaquero JM, Clemente-Postigo M, Estruch R, et al. Influence of red wine polyphenols and ethanol on the gut microbiota ecology and biochemical biomarkers. Am J Clin Nutr. 2012;95(6):1323-34.

47. Toral M, Robles-Vera I, De La Visitación N, Romero M, Yang T, Sánchez M, et al. Critical role of the interaction gut microbiota-sympathetic nervous system in the regulation of blood pressure. Front Physiol. 2019. https://doi. org/10.3389/fphys.2019.00231.

48. Yin X, Peng J, Zhao L, Yu Y, Zhang X, Liu P, et al. Structural changes of gut microbiota in a rat non-alcoholic fatty liver disease model treated with a Chinese herbal formula. Syst Appl Microbiol. 2013;36(3):188-96.

49. Beutler B. TLR4 as the mammalian endotoxin sensor. Curr Top Microbiol Immunol. 2002. https://doi.org/10.1007/978-3-642-59430-4_7.

50. Lamping N, Dettmer R, Schröder NWJ, Pfeil D, HallatschekW, Burger R, et al. LPS-binding protein protects mice from septic shock caused by LPS or gram-negative bacteria. J Clin Invest. 1998;101(10):2065-71.
51. Zhou CH, Meng YT, Xu JJ, Fang X, Zhao JL, Zhou W, et al. Altered diversity and composition of gut microbiota in Chinese patients with chronic pancreatitis: gut microbiota alterations in chronic pancreatitis. Pancreatology. 2020;20(1):16-24.

52. Bomfim GF, dos Santos RA, Oliveira MA, Giachini FR, Akamine EH, Tostes $\mathrm{RC}$, et al. Toll-like receptor 4 contributes to blood pressure regulation and vascular contraction in spontaneously hypertensive rats. Clin Sci. 2012;122(11):535-43.

53. Hernanz R, Martínez-Revelles S, Palacios R, Martín A, Cachofeiro V, Aguado A, et al. Toll-like receptor 4 contributes to vascular remodelling and endothelial dysfunction in angiotensin Il-induced hypertension. Br J Pharmacol. 2015;172(12):3159-76.

54. Conte MP, Schippa S, Zamboni I, Penta M, Chiarini F, Seganti L, et al. Gutassociated bacterial microbiota in paediatric patients with inflammatory bowel disease. Gut. 2006;55(12):1760-7.

55. Qin N, Yang F, Li A, Prifti E, Chen Y, Shao L, et al. Alterations of the human gut microbiome in liver cirrhosis. Nature. 2014;513(7516):59-64.

56. Koren O, Spor A, Felin J, Fåk F, Stombaugh J, Tremaroli V, et al. Human oral, gut, and plaque microbiota in patients with atherosclerosis. Proc Natl Acad Sci U S A. 2011;108(SUPPL. 1):4592-8.

57. Engels C, Ruscheweyh HJ, Beerenwinkel N, Lacroix C, Schwab C. The common gut microbe Eubacterium hallii also contributes to intestinal propionate formation. Front Microbiol. 2016. https://doi.org/10.3389/fmicb 2016.00713.

58. Duncan SH, Louis P, Flint HJ. Lactate-utilizing bacteria, isolated from human feces, that produce butyrate as a major fermentation product. Appl Environ Microbiol. 2004;70(10):5810-7.

59. Udayappan S, Manneras-Holm L, Chaplin-Scott A, Belzer C, Herrema H, Dallinga-Thie GM, et al. Oral treatment with Eubacterium hallii improves insulin sensitivity in db/db mice. NPJ Biofilms Microbiomes. 2016. https:// doi.org/10.1038/npjbiofilms.2016.9.

60. Zhang J, Song L, Wang Y, Liu C, Zhang L, Zhu S, et al. Beneficial effect of butyrate-producing Lachnospiraceae on stress-induced visceral hypersensitivity in rats. J Gastroenterol Hepatol. 2019;34(8):1368-76.

61. Groot HE, Van De VYJ, Verweij N, Lipsic E, Karper JC. Human genetic determinants of the gut microbiome and their associations with health and disease : a phenome - wide association study. Sci Rep. 2020. https://doi. org/10.1038/s41598-020-70724-5.

62. Calderón-pérez L, Gosalbes MJ, Yuste S, Valls RM, Pedret A, Llauradó E, et al. Gut metagenomic and short chain fatty acids signature in hypertension : a cross-sectional study. Sci Rep. 2020;10:1-16.

63. John OD, Mouatt P, Majzoub ME, Thomas T, Panchal SK, Brown L. Physiological and metabolic effects of yellow mangosteen (Garcinia dulcis) rind in rats with diet-induced metabolic syndrome. Int J Mol Sci. 2020;21(1):272.

64. Receptor P. Short-chain fatty acids stimulate angiopoietin-like 4 synthesis in human colon adenocarcinoma cells by activating peroxisome. Mol Cell Biol. 2013;33(7):1303-16.

65. Infirmary R. Role of anaerobic bacteria in the metabolic welfare of the colonic mucosa in man. Gut. 1980;21:793-8.

66. Donohoe DR, Garge N, Zhang X, Sun W, Connell TMO, Bunger MK, et al. Article The Microbiome and Butyrate Regulate Energy Metabolism and Autophagy in the Mammalian Colon. Cell Metab. 2011;13(5):517-26. https:// doi.org/10.1016/j.cmet.2011.02.018.

67. Duszka K, Oresic M, Le MC, König J. PPAR y Modulates Long Chain Fatty Acid Processing in the Intestinal Epithelium. Int J Mol Sci. 2017;18(12):2559.

68. Byndloss MX, Olsan EE, Rivera-chávez F, Tiffany CR, Cevallos SA, Lokken KL, et al. Microbiota-activated PPAR-g signaling inhibits dysbiotic Enterobacteriaceae expansion. Int J Hypertens. 2017;575(August):570-5.

69. Li J, Sun M, Ye J, Li Y, Zheng H, Liang F, et al. The mechanism of acupuncture in treating essential hypertension : a narrative review. Int J Hypertens. 2019;2019:8676490.

70. Rothschild D, Weissbrod O, Barkan E, Kurilshikov A, Korem T, Zeevi D, et al. Environment dominates over host genetics in shaping human gut microbiota. Nature. 2018;555(7695):210-5.

\section{Publisher's Note}

Springer Nature remains neutral with regard to jurisdictional claims in published maps and institutional affiliations. 